\title{
Development and validation of a chromatographic method for determining Clematichinenoside AR and related impurities
}

Yang Zhou, Yue Guan, Ji Shi, Xiaolin Zhang, Lan Yao and Lifang Liu*

\begin{abstract}
Background: Clematichinenoside AR is a promising lead compound for the treatment of rheumatoid arthritis. A systematic research for the related impurities in AR bulk samples is still lacking. For the safe use of this natural product in future clinical practice, the structure and content of each constituent, including the main ingredient as well as the impurities in AR bulk sample must be characterized in detail.
\end{abstract}

Results: A simple and stability indicating RP-HPLC method was developed and validated for determining the purity of clematichinenoside AR (AR), a natural product from the roots of Clematis manshurica Rupr. (Ranunculaceae) with the potential of treating rheumatoid arthritis. Five impurities were characterized, and impurity 2 (Clematomandshurica saponin F) is a new triterpenoid saponin isolated from this product. Optimum separation for clematichinenoside AR and five related impurities was carried out on an Agilent octadecylsilane bonded silica gel column (TC-C18, $4.6 \mathrm{~mm} \times 150 \mathrm{~mm}, 5 \mu \mathrm{m}$ ) using a gradient HPLC method. The validation results showed good sensitivity, specificity, linearity $\left(r^{2}>0.9992\right)$ precision( $\left.R S D<1.63 \%\right)$, accuracy(recoveries in the range of 95.60\%-104.76\%) and robustness. Three AR bulk samples containing all the impurities were examined by two methods, and the stability of correction factors for the determination of related impurities was discussed. The proposed stability-indicating method was suitable for the quality control of this natural product.

Conclusion: Five related impurities of clematichinenoside AR were characterized, including a new triterpenoid saponins firstly found in clematichinenoside AR bulk samples. In the simple chromatographic method for determining clematichinenoside AR and its related impurities in bulk samples, the correction factor was better for the quality control in the relative stable concentrations.

Keywords: Clematichinenoside AR, Related impurities, HPLC-UV, Correction factors

\section{Background}

Radix et Rhizoma Clematidis (Wei-Ling-Xian) has been used as an anti-inflammatory, antitumor, and analgesic agent in traditional Chinese medicine (TCM) with a long history [1,2]. Triterpenoid saponins are considered to be the main bioactive constituents in the root extracts of clematis species, and recent pharmacological studies revealed that some triterpenoid saponins have significant anti-inflammatory, antitumor and analgesic activities [3-7]. Clematichinenoside AR, 3-O- $\beta-[(\mathrm{O}-\alpha-\mathrm{L}-$ rhamnopyranosyl-( $1 \rightarrow 6)-\mathrm{O}-\beta-\mathrm{D}$-glucopyranosyl- $(1 \rightarrow 4)-\mathrm{O}-\beta$-D-glu-

\footnotetext{
* Correspondence: liulifang69@126.com

The State Key Laboratory of Natural Medicines, China Pharmaceutical University, Nanjing 210009, China
}

copyranosyl-( $(1 \rightarrow 4)$-O- $\beta$-D-ribopyranosyl- $(1 \rightarrow 3)$ - $\alpha$-OL-rhamnopyranosyl- $(1 \rightarrow 2)-\alpha$-L-arabinopyranosyl)oxy] oleanolic acid $28-\mathrm{O}-\alpha$-L-rhamnopyranosyl- $(1 \rightarrow 4)$ - $\beta$-D-glucopyranosyl-( $1 \rightarrow 6)-\beta$-D-glucopyranosyl ester, is a typical triterpenoid saponin isolated from the roots of Clematis chinensis Osbeck and Clematis manshurica Rupr. (Ranunculaceae). According to previous pharmacological studies, clematichinenoside AR has a potential anti-inflammatory effect in arthritic rats, and its mechanism may involve the inhibition of the expression of NF-kB p65 subunits, TNF-a and COX-2 [8-11]. In previous study, we established a HPLC-ELSD method to analyze triterpenoid saponins from Chinese clematis [12] and a LC-MS/MS method to detect clematichinenoside AR in rats after oral administration [13]. Our previous work revealed that clematichinenoside 
$\mathrm{AR}$ is a promising lead compound for the treatment of rheumatoid arthritis. For the safe use of this natural product in future clinical practice, the structure and content of each constituent, including the main ingredient as well as the impurities in AR bulk sample must be characterized in detail, as required by the State Food and Drug Administration (SFDA) in China. For this purpose, AR bulk samples were prepared using industry-scale production procedures with the AR content higher than 90\% (quantified by HPLC). Five related impurities were observed in chromatography, and the structure of the actual impurities that exceed $0.1 \%$ was characterized [14]. Besides, a new compound, clematomandshurica saponin F was isolated for the first time and confirmed as a new triterpenoid saponin by spectral methods (MS, ${ }^{1} \mathrm{H}$ NMR, ${ }^{13} \mathrm{C}$ NMR, COSY, DEPT, HMBC, HSQC and ROESY).

\section{Experimental}

\section{Materials and reagents}

All the chemical reference substances, including clematichinenoside AR and related substances were prepared in our laboratory, and the purity of each compound was confirmed to be higher than $98 \%$ by HPLC. Three batches of AR bulk samples were supplied by Chia Tai Tianqing Pharmaceutical Company. HPLC grade acetonitrile were purchased from Merck (Darmstadt, Germany), other analytical grade reagents were purchased from Nanjing Chemical Reagent Co., Ltd. (Nanjing, China).

\section{Apparatus and chromatographic conditions}

Analyses were primarily performed on an Agilent 1200 HPLC system (Agilent Technologies, CA, USA), equipped with a variable wavelength detector (VWD). The HPLC separation method was developed on an Agilent reversed phase octyldecyl silica column (TC-C18, $4.6 \mathrm{~mm} \times 150$ $\mathrm{mm}, 5 \mu \mathrm{m})$. The mobile phase consisted of solvent A (water) and solvent $\mathrm{B}$ (acetonitrile) with the following gradient program: $30 \% \mathrm{~B}$ in 0 5 min; 30\% 35\% B in 5 12 $\mathrm{min} ; 35 \% \sim 60 \% \mathrm{~B}$ in $12 \sim 17 \mathrm{~min} ; 60 \% \mathrm{~B}$ in $17 \sim 20 \mathrm{~min}$. (The flow rate was set at $1.0 \mathrm{~mL} / \mathrm{min}$, column temperature was kept at $30^{\circ} \mathrm{C}$ with the UV detection at $203 \mathrm{~nm}$ and the injection volume was $20 \mu \mathrm{L}$ ).

\section{Isolation and purification of related impurities}

For the isolation, a glass column with ODS material and semi-preparative HPLC instrument using an Agilent 1200 with Agilent Eclipse XDB-C18 $(9.4 \times 250$ mm, $5 \mu \mathrm{m}$, USA) were used to enrich and purify impurities. AR bulk sample (Lot: 20110610) dissolved in water was subjected to ODS column chromatography and eluted with gradient water-methanol. Each collected fraction was analyzed by TLC and HPLC. The fractions of the same compound were pooled together and purified by HPLC instrument. These impurities were used as reference substances in the following studies after structural elucidation by TOF-MS and NMR.

\section{Preparation of standard solutions}

AR reference standard solution $(2.0 \mathrm{mg} / \mathrm{mL})$ and five impurity solutions $(1.0 \mathrm{mg} / \mathrm{mL})$ were prepared respectively in a mixture of acetonitrile and water $(30: 70, \mathrm{v} / \mathrm{v})$ as standard stock solutions. The mixed standard solution was obtained by transferring the appropriate volumes of every stock solution into a $5 \mathrm{~mL}$ volumetric flask and diluting the mixture with the mobile phase in order to get standard solutions containing $1.0 \mathrm{mg} / \mathrm{mL} \mathrm{AR}$ and $0.05 \mathrm{mg} / \mathrm{mL}$ of each impurity (at $5.0 \%$ concentration relative to AR).

\section{Results and discussion}

\section{Structure elucidation of related impurities} Structure elucidation of impurity 2

Impurity 2 (Clematomandshurica saponin F) was a white, amorphous powder, and was freely dissolved in water and methanol, mp $231-232^{\circ} \mathrm{C}$. The optical rotation $\left([\mathrm{a}]_{\mathrm{D}}^{20}\right)$ was -38.1 (c $0.10,30 \%$ acetonitrile). The IR spectrum showed the presence of hydroxyl groups at $3425 \mathrm{~cm}^{-1}$ and carbonyl groups at $1640 \mathrm{~cm}^{-1}$.

HR-ESI MS gave a strong molecular ion with the $\mathrm{m} / \mathrm{z}$ value of 1821.8175 , suggesting the molecular formula as $\mathrm{C}_{82} \mathrm{H}_{134} \mathrm{O}_{44}$. The NMR spectra were recorded at $303 \mathrm{k}$ on a Bruker AV-500 NMR (1H NMR, $500 \mathrm{MHz}$; 13C NMR, $125 \mathrm{MHz}$ ) instrument using pyridine-d5.

The ${ }^{13} \mathrm{C}$ NMR spectral data reported in Table 1 are in agreement with those in the literature [15]. More details of the structure were elucidated by analyzing the ${ }^{1} \mathrm{H}$ and ${ }^{13} \mathrm{C}$ NMR spectra. The ${ }^{13} \mathrm{C}$ NMR spectrum showed 82 carbon signals. There were 28 signals at upfield ( $\delta 0-60)$, in which 25 signals were due to aglycone and three signals from C-6 of the three rhamnose $(\delta 18.4,18.5,18.6) .39$ signals were shown from $\delta 60$ to $\delta 90$, in which $\delta 81.9$ and $\delta 63.9$ were from the aglycone. 9 signals exhibited from $\delta$ 90 to $\delta 100$ ( $\delta$ 95.6, 101.4, 102.7, 102.7, 103.2, 104.6, 104.7, $104.8,104.9)$ were signals of the sugar anomeric carbons. There were two signals showed from $\delta 110$ to $\delta 150$, which were from the aglycone. The signal at $\delta 176.5$ was due to carbonyl group. The ${ }^{13} \mathrm{C}$ NMR spectral data reported in Table 1 are in agreement with those in the literature [15], suggesting the hederagenin as the aglycone. The ${ }^{1} \mathrm{H}$ NMR spectrum exhibited nine signals of the sugar anomeric protons at $\delta 5.04(1 \mathrm{H}, \mathrm{m}, \mathrm{H}-1$ of Ara), 6.25 (1H, brs, H-1 of Rha), 5.80(1H, brs, H-1 of Rib), 4.91 $(1 \mathrm{H}, \mathrm{m}, \mathrm{H}-1$ of Glc), 5.07(1H, d, H-1 of Glc'), 5.40(1H, brs, H-1 of Rha'), $6.20(1 \mathrm{H}, \mathrm{d}, \mathrm{H}-1$ of Glc"), $4.97(1 \mathrm{H}, \mathrm{d}, \mathrm{H}-1$ of Glc"'), $5.81(1 \mathrm{H}, \mathrm{s}, \mathrm{H}-1$ of Rha") and methyl signals of the three rhamnose at $\delta 1.67(3 \mathrm{H}, \mathrm{d}, \mathrm{J}=6.1 \mathrm{~Hz}, \mathrm{Me}-6$ of Rha"), $1.57(3 \mathrm{H}, \mathrm{d}, \mathrm{J}=6.0 \mathrm{~Hz}, \mathrm{Me}-6$ of Rha'), $1.51(3 \mathrm{H}, \mathrm{d}, \mathrm{J}=6.0 \mathrm{~Hz}$, Me- 6 of Rha). Also there are six methyl signals of aglycone shown in the ${ }^{1} \mathrm{H}$ NMR spectrum at $\delta 1.15(3 \mathrm{H}, \mathrm{s}, \mathrm{Me}-27)$, 
Table $1{ }^{1} \mathrm{H}$ and ${ }^{13} \mathrm{C}$ NMR spectra data of clematomandshurica saponin $F$ (pyridine-d5)

\begin{tabular}{|c|c|c|}
\hline Position & $\delta_{\mathrm{C}} / \mathrm{ppm}$ & $\delta_{\mathrm{H}} / \mathrm{ppm}$ \\
\hline 1 & 39.13 & $1.06,1,51$ \\
\hline 2 & 26.39 & $1.99,2.20$ \\
\hline 3 & 81.93 & 4.13 \\
\hline 4 & 43.62 & \\
\hline 5 & 47.73 & 1.67 \\
\hline 6 & 18.17 & $1.26,1.28$ \\
\hline 7 & 32.78 & $1.51,1.52$ \\
\hline 8 & 39.94 & \\
\hline 9 & 48.24 & 1.71 \\
\hline 10 & 36.92 & \\
\hline 11 & 23.39 & 1.88 \\
\hline 12 & 123.72 & 5.37 \\
\hline 13 & 144.12 & \\
\hline 14 & 42.15 & \\
\hline 15 & 28.31 & 2.20 \\
\hline 16 & 23.85 & 1.99 \\
\hline 17 & 47.05 & \\
\hline 18 & 41.67 & $3.12,3.15$ \\
\hline 19 & 46.22 & $1.19,1.67$ \\
\hline 20 & 30.74 & \\
\hline 21 & 34.03 & 1.28 \\
\hline 22 & 32.57 & $1.70,1.85$ \\
\hline 23 & 63.96 & $4.24,3.89, \mathrm{CH}_{2} \mathrm{OH}$ \\
\hline 24 & 14.09 & $1.10,5$ \\
\hline 25 & 16.21 & $0.94,5$ \\
\hline 26 & 17.56 & $1.06,5$ \\
\hline 27 & 26.07 & $1.15, \mathrm{~s}$ \\
\hline 28 & 176.51 & \\
\hline 29 & 33.11 & $0.85,5$ \\
\hline \multirow[t]{2}{*}{30} & 23.72 & $0.87, \mathrm{~s}$ \\
\hline & 3-o-sugar & \\
\hline Ara 1 & 104.95 & 5.04 \\
\hline 2 & 75.18 & 4.52 \\
\hline 3 & 74.77 & 4.24 \\
\hline 4 & 69.28 & 4.28 \\
\hline 5 & 66.25 & $3.65,4.22$ \\
\hline Rha 1 & 101.41 & 6.25, brs \\
\hline 2 & 71.95 & 4.82 \\
\hline 3 & 82.02 & 4.67 \\
\hline 4 & 72.77 & 4.37 \\
\hline 5 & 69.87 & 4.63 \\
\hline 6 & 18.45 & $1.51, \mathrm{~d}, 6.0 \mathrm{~Hz}$ \\
\hline
\end{tabular}

Table $1{ }^{1} \mathrm{H}$ and ${ }^{13} \mathrm{C}$ NMR spectra data of clematomandshurica saponin $\mathbf{F}$ (pyridine-d5) (Continued)

\begin{tabular}{|c|c|c|}
\hline Rib 1 & 104.63 & $5.80, \mathrm{brs}$ \\
\hline 2 & 72.64 & 4.38 \\
\hline 3 & 69.71 & 4.54 \\
\hline 4 & 76.55 & 3.85 \\
\hline 5 & 61.97 & 4.40 \\
\hline Glc 1 & 103.22 & $4.91, \mathrm{~m}$ \\
\hline 2 & 74.14 & 4.28 \\
\hline 3 & 76.69 & 4.28 \\
\hline 4 & 81.05 & 4.63 \\
\hline 5 & 75.36 & 3.91 \\
\hline 6 & 61.75 & 4.20 \\
\hline Glc' 1 & 104.85 & $5.07, \mathrm{~d}, 8.1 \mathrm{~Hz}$ \\
\hline 2 & 74.24 & 3.83 \\
\hline 3 & 78.37 & 4.16 \\
\hline 4 & 71.95 & 4.70 \\
\hline 5 & 76.80 & 4.02 \\
\hline 6 & 68.57 & $3.92,4.60$ \\
\hline Rha' 1 & 102.74 & $5.40, \mathrm{brs}$ \\
\hline 2 & 71.75 & 4.68 \\
\hline 3 & 72.56 & 4.52 \\
\hline 4 & 73.89 & 4.18 \\
\hline 5 & 69.75 & 4.27 \\
\hline \multirow[t]{2}{*}{6} & 18.52 & $1.57, \mathrm{~d}, 6.0 \mathrm{~Hz}$ \\
\hline & 28-o-sugar & \\
\hline Glc" 1 & 95.65 & $6.20, \mathrm{~d}, 8.0 \mathrm{~Hz}$ \\
\hline 2 & 73.83 & 4.07 \\
\hline 3 & 78.75 & 4.14 \\
\hline 4 & 70.95 & 4.24 \\
\hline 5 & 78.05 & 4.07 \\
\hline 6 & 69.28 & 4.62 \\
\hline Glc" 1 & 104.71 & $4.97, \mathrm{~d}, 7.8 \mathrm{~Hz}$ \\
\hline 2 & 74.85 & 3.88 \\
\hline 3 & 76.40 & 3.85 \\
\hline 4 & 78.22 & 4.38 \\
\hline 5 & 77.16 & 3.63 \\
\hline 6 & 61.35 & 4.07 \\
\hline Rha" 1 & 102.76 & 5.81 \\
\hline 2 & 72.56 & 4.07 \\
\hline 3 & 72.83 & 4.50 \\
\hline 4 & 74.09 & 3.95 \\
\hline 5 & 70.32 & 4.91 \\
\hline 6 & 18.62 & $1.67, \mathrm{~d}, 6.1 \mathrm{~Hz}$ \\
\hline
\end{tabular}


1.10(3H, s, Me-24), 1.06(3H, s, Me-26), 0.94(3H, s, Me25), 0.87(3H, s, Me-30), 0.85(3H, s, Me-29). The downfield shift of $\mathrm{C}-3$ and the upfield shift of $\mathrm{C}-28$ suggested that the hydroxyl group of $\mathrm{C}-3$ and the carbonyl group of $\mathrm{C}-28$ were both glycosydated. The chemical shift of C-23 moved to the downfield at $\delta 63.96$ because of the hydroxylation. The acid hydrolysis showed the presence of arabinose, glucose, rhamnose and ribose. Compared with the data in the literature [5], the exact sugar sequence and its linkage position to the aglycone were solved by a detailed analysis of the 2D NMR spectra. The HMBC spectrum showed the correlations from H-Rha'-1 to C-Glc'-6, H-Ara-1 to C-3; H-Ara-2 to C-Rha-1; H-Rha-1 to C-Ara-2; H-Rha-3 to C-Rib-1; H-Glc-1 to C-Rib-4; H-Glc'-1 to C-Glc-4; H-Glc"-1 to C-28; H-Glc"'-1 to C-Glc"-6; H-Rha"-1 to C-Glc"'-4. Based on these analyses, the structure of this compound was elucidated as $3-\mathrm{O}-\beta-[(\mathrm{O}-\alpha-\mathrm{L}-\mathrm{rhamno}-$ pyranosyl- $(1 \rightarrow 6)-O-\beta$-D-glucopyranosyl- $(1 \rightarrow 4)-O-\beta-D-$ glucopyranosyl- $(1 \rightarrow 4)-\mathrm{O}-\beta-\mathrm{D}$-ribopyranosyl- $(1 \rightarrow 3)-\mathrm{O}-$ $\alpha$-L-rhamnopyranosyl- $(1 \rightarrow 2) \quad-\alpha$-L-arabinopyranosyl)oxy] hederagenin 28-O- $\alpha-\mathrm{L}$ - rhamnopyranosyl- $(1 \rightarrow 4)-\mathrm{O}-\beta-\mathrm{D}-$ glucopyanosyl- $(1 \rightarrow 6)-\beta$-D-glucopyanosyl ester. The spectra data assignments were shown in Table 1.

\section{Structure elucidation of impurities 1, 3, 4, 5}

Impurities 1, 3, 4, 5 were obtained as white, amorphous powders. The TOF-MS revealed impurities 1, 3, 4 have double charged molecular ion at $\mathrm{m} / \mathrm{z}$ 933.4001, 852.3874, 794.3646, and gave impurity 5 has molecular ion at $\mathrm{m} / \mathrm{z}$ 1335.6913, which corresponds to a molecular formula of $\mathrm{C}_{82} \mathrm{H}_{134} \mathrm{O}_{44}, \mathrm{C}_{76} \mathrm{H}_{124} \mathrm{O}_{39}, \mathrm{C}_{69} \mathrm{H}_{124} \mathrm{O}_{34}$, and $\mathrm{C}_{64} \mathrm{H}_{104} \mathrm{O}_{29}$, respectively. Their chemical structures were suggested as clematichinenoside $\mathrm{AR}_{6}$ [3], clematomandshurica saponin C [4], clematichinenoside $C$ [16], and clematichinenoside $\mathrm{AR}_{2}$ [5] by comparing their mass spectral data with those published in the literature. The structures of these related substances were further confirmed by the same retention time in HPLC chromatogram with each of their reference substances shown above. The chemical structures of these related substances are shown in Figure 1.

\section{Development and validation of HPLC method}

A simple, efficient and reliable method was developed to examine AR and five impurities in bulk samples. AR and its related impurities all showed maximum absorption at $203 \mathrm{~nm}$. Therefore, the detection wavelength was set at $203 \mathrm{~nm}$, flow rate and column temperature were optimized in order to achieve the desired resolution and tailing factor of closely eluting impurities using simple chromatographic conditions. Under this condition, all the peaks were clearly separated. The proposed method was validated with respect to system suitability, specificity, robustness, linearity, accuracy, precision, LOD and LOQ. Figure 2 showed a typical chromatogram of AR and five potential impurities. Good peak symmetries and resolutions were observed for AR and all spiked impurity components in $20 \mathrm{~min}$. Results were summarized in Table 2.

\section{Effect of three C18 columns}

To investigate the effect of the HPLC column on the separation and tailing factor under the optimized conditions, octyldecyl silica gel columns from three different vendors were evaluated for the analysis of AR and its impurities. These HPLC columns included: (1) Thermo ODS Hypersil (4.6 mm $\times 150 \mathrm{~mm}, 5 \mu \mathrm{m}$, USA); (2)Diamonsil C18(4.6 mm $\times 150 \mathrm{~mm}, 5 \mu \mathrm{m}$, China) (3) Agilent TC-C18(4.6mm $\times 150 \mathrm{~mm}, 5 \mu \mathrm{m}$, USA). Based on the results from the laboratory HPLC system, all the resolutions were more than 1.50, but the Agilent TC-C18 provided the best resolutions. The tailing factors of AR and impurities obtained on the Agilent TC18 column were between 0.8 and 1.2 , which indicated good peak shapes for all compounds. Tailing factors of Imp-1, 5 on the Diamonsil C18 column were less than 0.8 and the numbers of theoretical plates for Imp-2, 3, 4 and AR were less than those obtained on the Agilent TC-C18. Besides, the tailing factors of Imp-1, 5 were bigger than 1.5 and was not suitable for tailing factor requirement on the Thermo ODS Hypersil. Thus, the Agilent TC-C18 column demonstrated the desirable performance and was selected for further studies. The results were listed in Table 3.

\section{System suitability}

The system suitability tests were conducted using the working solution containing AR $(1.0 \mathrm{mg} / \mathrm{mL})$ and five impurities (each at $0.05 \mathrm{mg} / \mathrm{mL}$ ) by five repeated injections with the optimized method. It was observed that the tailing factors for all compounds were between 0.8 and 1.2, and the resolutions were greater than 1.50 . These results met the HPLC method requirements for separation and quantification of AR and related impurities in bulk samples.

\section{Specificity}

The specificity of the related compounds and AR was assessed using the forced degradation studies under conditions described in the literature [17]. Basic, acidic, oxidative, photolytic and thermal degradation were conducted in $0.1 \mathrm{~N} \mathrm{NaOH}$ at $60^{\circ} \mathrm{C}$ for $40 \mathrm{~min}, 1 \mathrm{~N} \mathrm{HCl}$ at $60^{\circ} \mathrm{C}$ for $30 \mathrm{~min}, 3 \% \mathrm{H}_{2} \mathrm{O}_{2}$ at $60^{\circ} \mathrm{C}$ for $1 \mathrm{~h}$, illumination of 1.2 million lux hours for 10 days and heat at $60^{\circ} \mathrm{C}$ for $8 \mathrm{~h}$, respectively. Photolytic and thermal studies were conducted by exposing the samples both in solution $(1.0 \mathrm{mg} / \mathrm{mL})$ and in solid state. Samples were withdrawn at the appropriate times, the $\mathrm{pH}$ was adjusted to neutral and the samples were subjected to HPLC analysis after suitable dilution $(1.0 \mathrm{mg} / \mathrm{mL})$ to evaluate the ability of the 


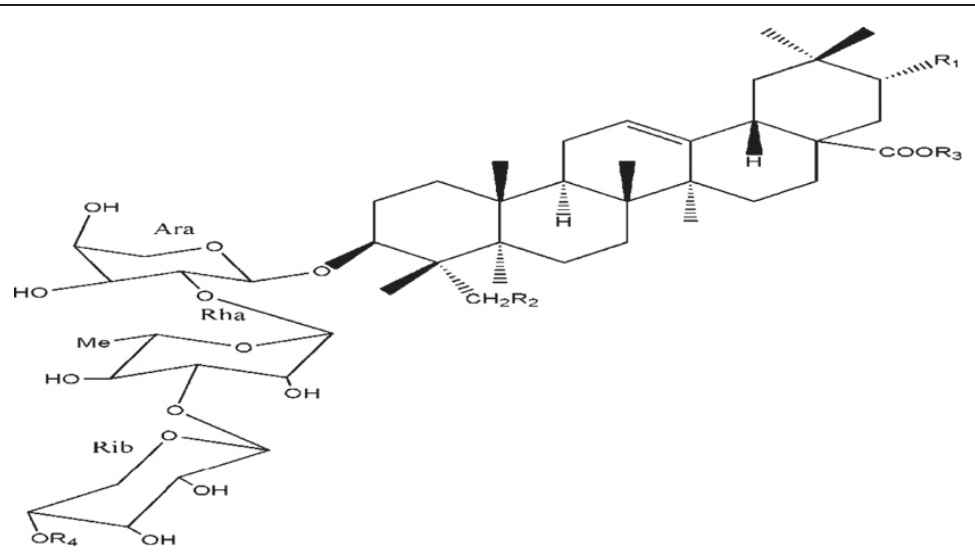

\begin{tabular}{lllll}
\hline & R1 & R2 & R3 & R4 \\
\hline Clematichinenoside & H & H & S1 & S3
\end{tabular}

AR

\begin{tabular}{lllll} 
Imp-1 & $\mathrm{OH}$ & $\mathrm{H}$ & $\mathrm{S} 1$ & $\mathrm{~S} 3$ \\
Imp-2 & $\mathrm{H}$ & $\mathrm{HO}$ & $\mathrm{S} 1$ & $\mathrm{~S} 3$ \\
Imp-3 & $\mathrm{H}$ & $\mathrm{H}$ & $\mathrm{S} 1$ & $\mathrm{~S} 2$ \\
Imp-4 & $\mathrm{H}$ & $\mathrm{H}$ & $\mathrm{S} 1$ & $\mathrm{~S} 4$ \\
Imp-5 & $\mathrm{H}$ & $\mathrm{H}$ & $\mathrm{H}$ & $\mathrm{S} 3$ \\
\hline
\end{tabular}

$\mathbf{S}_{1}$

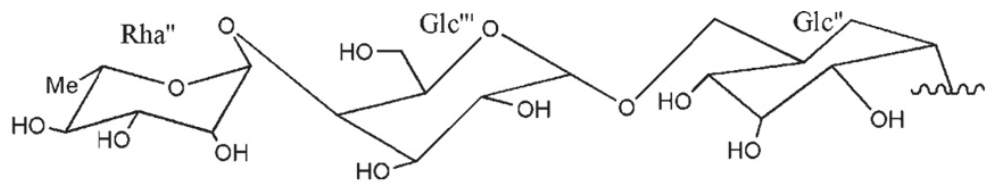

$S_{2}$

S3
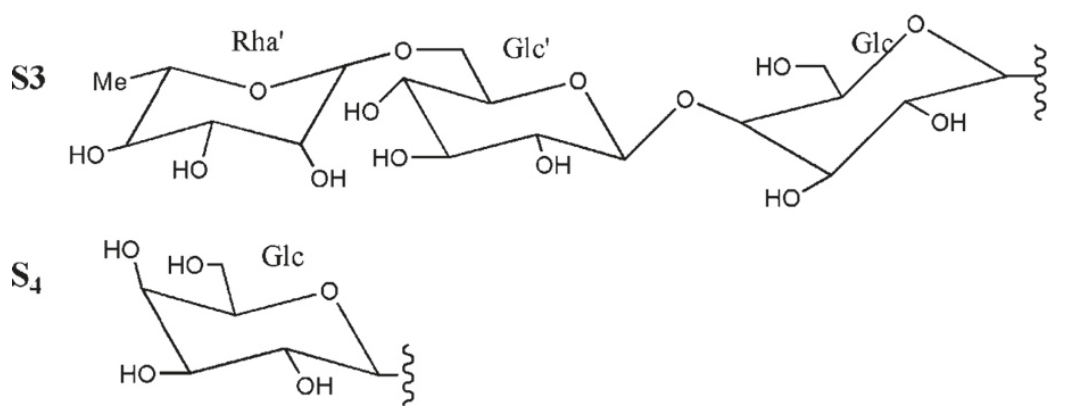

Figure 1 The chemical structures of Clematichinenoside AR and its related impurities. 


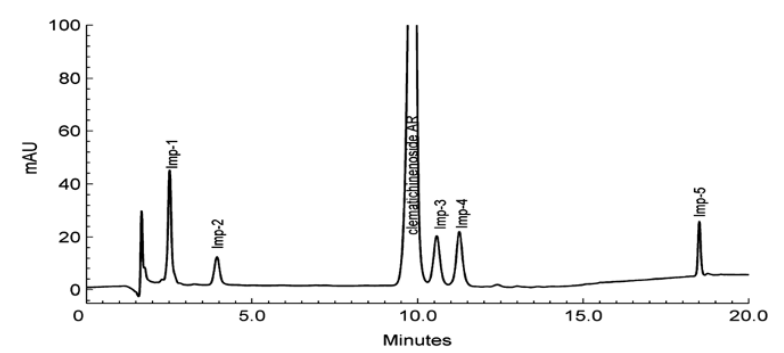

Figure 2 A typical chromatogram of clematichinenoside AR bulk sample.

proposed method to separate AR from its potential impurities. The bulk drug and degradation samples were examined by peak purity testing utilizing DAD detector. The purity factor obtained from AR peak was higher than threshold, which demonstrated the spectral homogeneity. Degradation of the sample at $60^{\circ} \mathrm{C}$ and illumination of 1.2 million lux hours did not show any difference in terms of chromatographic behavior compared to a fresh one, but in the acid and basic conditions, the content of Imp- 5 increased significantly. Besides, clematichinenoside AR was transformed into Imp-4 in acidic condition. All the results demonstrated that degradation products formed during the stress studies were well separated from AR which proved that the adopted method was specific (Figure 3).

\section{Precision}

The method reproducibility was evaluated by determining precision on a spiked AR solution $(1.0 \mathrm{mg} / \mathrm{mL})$ with each impurity at $5.0 \%$ concentration $(0.05 \mathrm{mg} / \mathrm{mL})$. Each RSD (\%) of peak areas for all components was below $2.0 \%$ as shown in Table 2, suggesting good method reproducibility.

\section{$\angle O D$ and $L O Q$}

The standard solutions containing both AR and impurities were injected and the peak height of each component was recorded. The limit of detection (LOD) and limit of quantitation (LOQ) for AR and five impurities were calculated by normalization of the signal/noise ratio $(\mathrm{S} / \mathrm{N})$ to 3 for LOD and $\mathrm{S} / \mathrm{N}$ to 10 for $\mathrm{LOQ}$, see Table 2. The RSD for LOQ concentrations for all compounds was below $5.0 \%$.

\section{Linearity}

The linearity of test solution was prepared at six concentration levels, 0.01 to $2.0 \mathrm{mg} / \mathrm{mL}$ for $\mathrm{AR}$, from 6 to $60 \mu \mathrm{g} \cdot \mathrm{ml}^{-1}$ for impurity 2 and 1 , from 10 to $100 \mu \mathrm{g} \cdot \mathrm{ml}^{-1}$ for impurities 3, 4 and 5. The adopted method demonstrated good linearity for clematichinenoside AR $\left(r^{2}>0.9994\right)$ and five impurity components $\left(r^{2}>0.9992\right)$.

\section{Accuracy}

The accuracy of the method was checked for three known concentration levels $(80 \%, 100 \%, 120 \%)$ added to a sample containing all compounds and peak area was

Table 2 Method validation data for clematichinenoside AR and its related impurities

\begin{tabular}{|c|c|c|c|c|c|c|}
\hline Parameters & Imp-1 & Imp-2 & AR & Imp-3 & Imp-4 & Imp-5 \\
\hline \multicolumn{7}{|l|}{ System suitability } \\
\hline$R T^{b}$ & $2.533 \pm 0.008$ & $3.934 \pm 0.016$ & $9.793 \pm 0.041$ & $10.518 \pm 0.037$ & $11.206 \pm 0.037$ & $18.449 \pm 0.011$ \\
\hline$R R T^{b, c}$ & $0.259 \pm 0.0003$ & $0.402 \pm 0.0002$ & $1.000 \pm 0.0000$ & $1.074 \pm 0.0008$ & $1.144 \pm 0.0012$ & $1.884 \pm 0.0071$ \\
\hline$R_{S}^{b, c}$ & & $6.79 \pm 0.050$ & $18.33 \pm 0.175$ & $1.99 \pm 0.008$ & $2.06 \pm 0.008$ & $26.91 \pm 0.381$ \\
\hline$A_{S}^{b, c}$ & $1.124 \pm 0.004$ & $1.047 \pm 0.057$ & $0.838 \pm 0.003$ & $0.964 \pm 0.003$ & $0.974 \pm 0.004$ & $1.012 \pm 0.0091$ \\
\hline$a^{b, c}$ & & $1.872 \pm 0.434$ & $1.872 \pm 0.434$ & $1.080 \pm 0.008$ & $1.070 \pm 0.006$ & $1.686 \pm 0.056$ \\
\hline$K^{\prime} b, c$ & $0.693 \pm 0.005$ & $1.630 \pm 0.011$ & $5.547 \pm 0.028$ & $6.032 \pm 0.025$ & $6.492 \pm 0.025$ & $11.335 \pm 0.007$ \\
\hline NTP b, c & $4009 \pm 44$ & $3889 \pm 107$ & $10299 \pm 174$ & $14992 \pm 321$ & $19418 \pm 130$ & $248207 \pm 2222$ \\
\hline \multicolumn{7}{|l|}{ Linearity } \\
\hline$r^{2}$ & 0.9998 & 0.9994 & 0.9994 & 0.9998 & 0.9994 & 0.9992 \\
\hline Slope & 2.9525 & 2.9072 & 2.9442 & 3.1068 & 3.5708 & 4.9601 \\
\hline Intercept & -0.0324 & -2.3100 & 0.2528 & -0.9281 & -2.0499 & 0.3703 \\
\hline Precision(RSD n=6,\%) & 0.76 & 1.04 & 0.22 & 0.76 & 0.45 & 1.63 \\
\hline $\mathrm{LOD}(\mu \mathrm{g} \cdot \mathrm{ml}-1)$ & 0.55 & 1.87 & 0.49 & 0.57 & 0.53 & 1.04 \\
\hline $\mathrm{LOQ}(\mu \mathrm{g} \cdot \mathrm{ml}-1)$ & 1.10 & 2.80 & 0.99 & 1.13 & 1.06 & 2.08 \\
\hline Accuracy (80\%) & $99.88 \%$ & $99.54 \%$ & $98.73 \%$ & $100.29 \%$ & $95.60 \%$ & $104.76 \%$ \\
\hline Accuracy (100\%) & $99.30 \%$ & $100.36 \%$ & $99.44 \%$ & $100.23 \%$ & $99.56 \%$ & $96.15 \%$ \\
\hline Accuracy(120\%) & $101.23 \%$ & $96.50 \%$ & $103.56 \%$ & $102.67 \%$ & $98.08 \%$ & $98.08 \%$ \\
\hline
\end{tabular}

$R T$, retention time/min; $R R T$, relative retention time; $R$, resolution; $A$, tailing factor; $a$, selectivity; $K$, capacity; NTP, number of theoretical plates. ${ }^{\mathrm{b}}$ Average of five determinations. ${ }^{\mathrm{C}}$ Mean $\pm \mathrm{SD}(\mathrm{n}=5)$. 
Table 3 Comparison of three columns under optimized conditions

\begin{tabular}{|c|c|c|c|c|c|c|}
\hline Columns & Compounds & Rs & As & $a$ & $\mathbf{k}^{\prime}$ & NTP \\
\hline Thermo ODS Hypersil & Imp-1 & - & 1.681 & - & 0.279 & 8610 \\
\hline \multirow[t]{5}{*}{$(4.6 \mathrm{~mm} \times 150 \mathrm{~mm}, 5 \mu \mathrm{m})$} & $\operatorname{Imp}-2$ & 3.62 & 1.281 & 1.234 & 0.579 & 3280 \\
\hline & $A R$ & 9.49 & 0.879 & 2.583 & 2.178 & 3162 \\
\hline & Imp-3 & 1.60 & 1.036 & 1.119 & 2.559 & 3255 \\
\hline & Imp-4 & 1.63 & 0.934 & 1.116 & 2.979 & 3615 \\
\hline & Imp-5 & 40.03 & 2.778 & 3.215 & 10.974 & 127412 \\
\hline \multirow[t]{6}{*}{ Diamonsil C18(4.6mm ×150mm, $5 \mu \mathrm{m})$} & Imp-1 & - & 0.707 & - & 0.237 & 5722 \\
\hline & Imp-2 & 3.37 & 1.135 & 1.283 & 0.586 & 2009 \\
\hline & $A R$ & 9.41 & 0.878 & 3.051 & 2.786 & 2167 \\
\hline & Imp-3 & 1.52 & 0.977 & 1.135 & 3.297 & 2448 \\
\hline & Imp-4 & 1.90 & 0.951 & 1.157 & 3.977 & 2948 \\
\hline & Imp-5 & 27.88 & 0.691 & 2.341 & 10.740 & 159346 \\
\hline \multirow[t]{6}{*}{ Agilent TC-C18(4.6mm×150mm,5 $\mu \mathrm{m})$} & Imp-1 & - & 1.124 & - & 0.693 & 4009 \\
\hline & Imp-2 & 6.79 & 1.047 & 1.872 & 1.630 & 3889 \\
\hline & $A R$ & 18.33 & 0.838 & 2.856 & 5.547 & 10299 \\
\hline & Imp-3 & 1.99 & 0.964 & 1.080 & 6.032 & 14992 \\
\hline & Imp-4 & 2.06 & 0.974 & 1.070 & 6.492 & 19418 \\
\hline & Imp-5 & 26.91 & 1.012 & 1.686 & 11.335 & 248207 \\
\hline
\end{tabular}

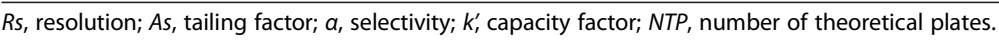

recorded. The mean analyte recoveries and their recovery rates obtained for each level are described in detail in Table 2 . The accuracy and recovery results were suitable for the quantitative HPLC method.

\section{Robustness}

For an analytical method to be robust, it must be able to produce quantitative results despite small changes in the experimental parameters, which may occur in a typical testing laboratory. The robustness of the method was studied by employing an experimental design, where the temperature of the column $\left(25^{\circ} \mathrm{C}-35^{\circ} \mathrm{C}\right)$ and the flow rate of the mobile phase $\left(0.8-1.2 \mathrm{~mL} \cdot \mathrm{min}^{-1}\right)$ were purposely subjected to small modifications. The temperature, the flow rate and the combined effects as the three factors were studied on the characteristics of the chromatogram of AR and its impurities. The tailing factors for all compounds were between 0.8 and 1.2 and the resolutions were greater than 1.50 .

\section{Application of correction factors Calculation of correction factor}

Due to the difficulties and expenses to prepare all the related reference standards, the application of the external standard method was limited, so correction factor was used in the quantification of related substances. According to $\mathrm{ICH}$ guiding principles, if the response factors of drug substance and relevant impurity are not close, we can use the drug substance as a standard to estimate the levels of impurities with providing a correction factor. The method of calculating correction factor is the same as conversion factor [18]. The correction factor (f) was the ratio of responses between internal standard substance (As/Cs) and standard substance $\left(A_{R} / C_{R}\right)$.

$$
f=\frac{\mathrm{A}_{\mathrm{s}} / \mathrm{C}_{\mathrm{s}}}{\mathrm{A}_{\mathrm{R}} / \mathrm{C}_{\mathrm{R}}}
$$

As: peak area of internal standard substance; Cs: concentration of internal standard substance; $A_{R}$ : peak area of standard substance; Cs: concentration of standard substance

In this study, AR was selected as internal standard, and each related impurity was as standard. The correction factor of each substance was obtained as the mean values calculated from five different concentrations. The results from five gradient concentrations showed that the correction factors of Imp-1 (F1) was $1.00 \pm 0.04$, the Imp-2 (F2) was $1.13 \pm 0.02$, the Imp-3 (F3) was $1.00 \pm 0.02$, the Imp-4 (F4) was $0.90 \pm 0.04$, and the Imp-5 (F5) was $0.61 \pm 0.02$. The RSDs for corrections for all compounds were below $5 \%$. Additionally, there were other two approaches used to calculate the correction factors. The first one was calculated by the ratio of equation slope of $\mathrm{AR}$ and its impurities. F1 was 1.00; F2 was 1.01; F3 was 0.95; F4 was 0.82 and F5 was 0.59 . The above results showed that F1 and F5 were similar 
(a)

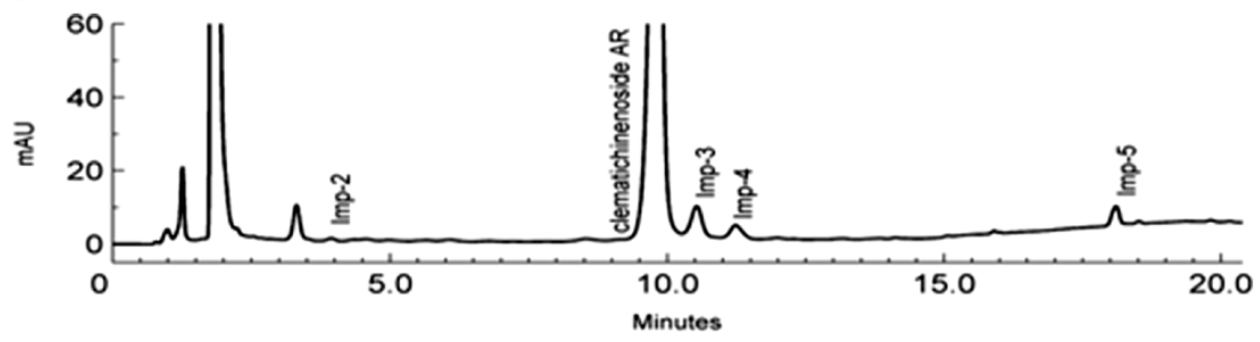

(b)

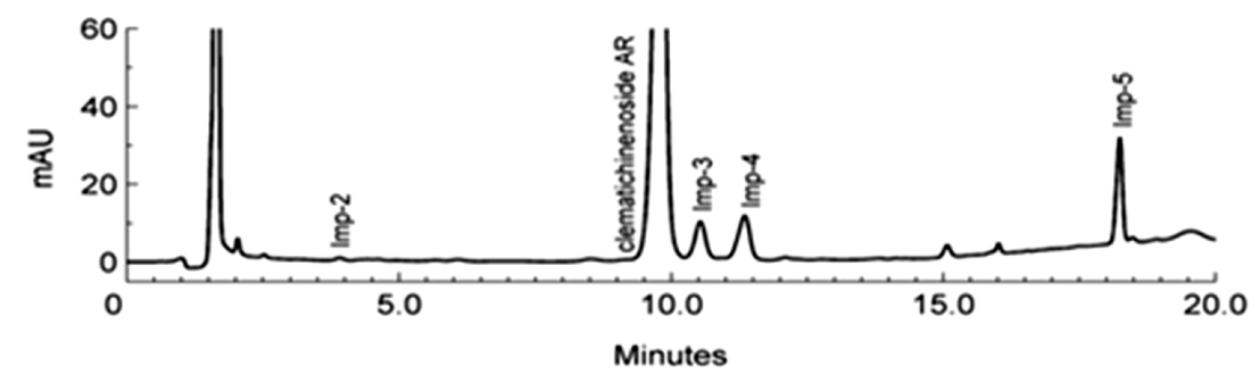

(c)

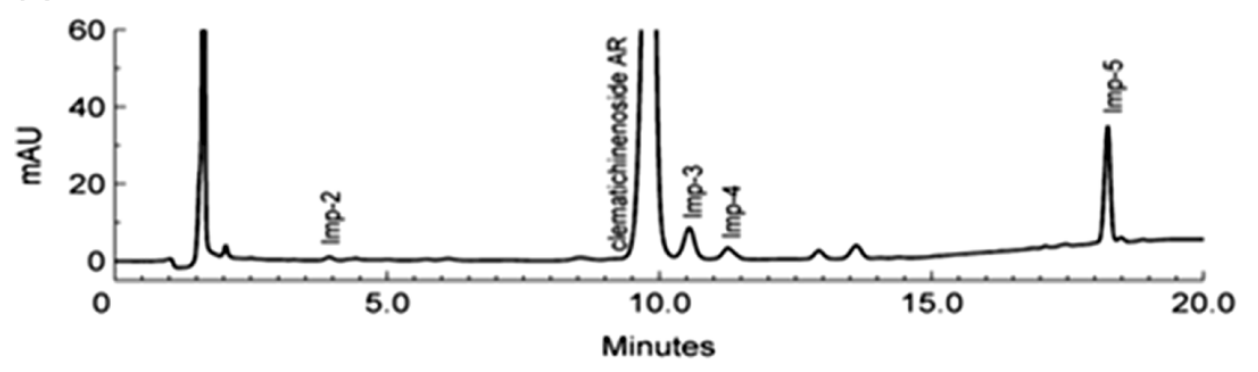

(d)

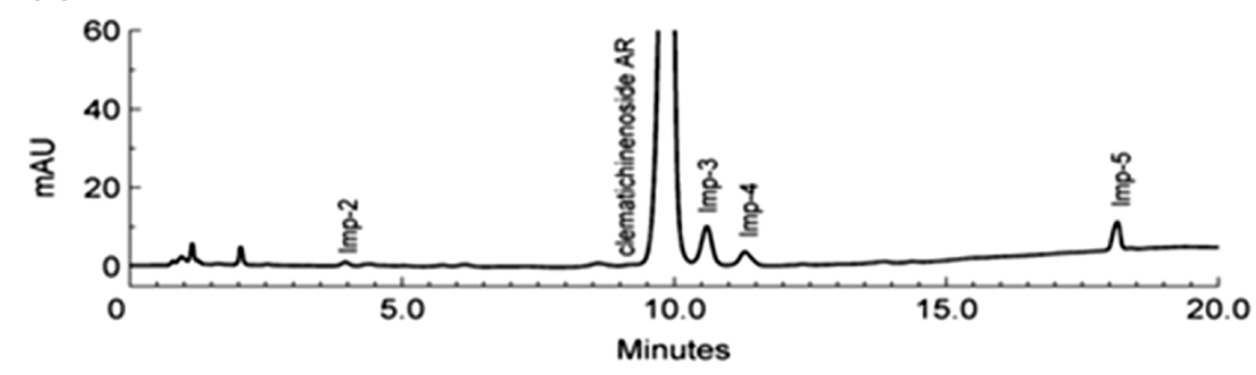

(e)

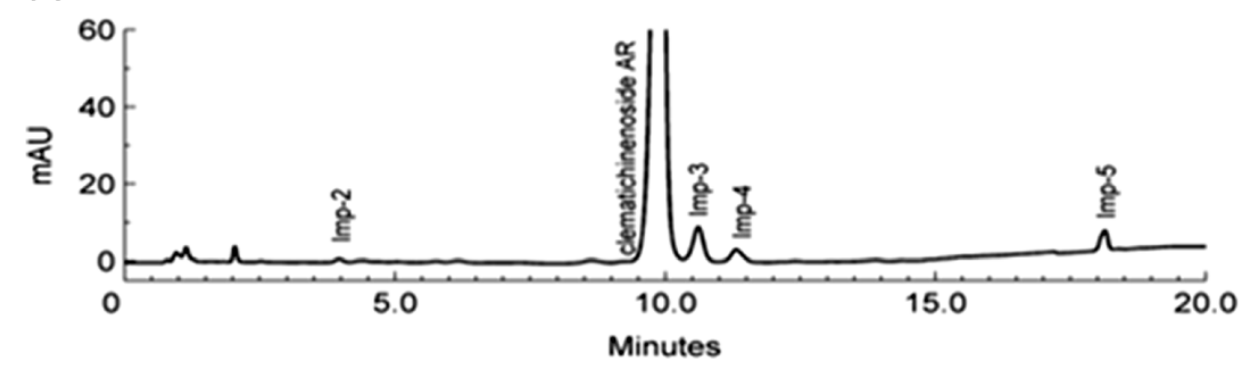

Figure 3 Typical chromatogram of clematichinenoside AR under stress conditions: (a) oxidative degradation, (b) acid hydrolysis, (c) base hydrolysis, (d) thermal degradation, (e) photolytic degradation. 
Table 4 Correction factors of related impurities

\begin{tabular}{|c|c|c|c|c|c|c|c|c|c|c|c|c|}
\hline & $\operatorname{Imp}-1(\mu \mathrm{g} / \mathrm{mL})$ & F1 & $\operatorname{Imp}-2(\mu \mathrm{g} / \mathrm{mL})$ & F2 & $A R(\mu \mathrm{g} / \mathrm{mL})$ & Fo & $\operatorname{Imp}-3(\mu \mathrm{g} / \mathrm{mL})$ & F3 & Imp-4( $\mu \mathrm{g} / \mathrm{mL})$ & F4 & $\operatorname{Imp}-5(\mu \mathrm{g} / \mathrm{mL})$ & F5 \\
\hline 1 & 6.41 & 0.96 & 6.26 & 1.15 & 9.52 & 1 & 9.83 & 1.01 & 6.50 & 0.94 & 5.98 & 0.60 \\
\hline 2 & 12.82 & 0.97 & 12.53 & 1.13 & 19.05 & 1 & 19.66 & 1.02 & 13.01 & 0.91 & 11.95 & 0.60 \\
\hline 3 & 26.70 & 1.00 & 26.10 & 1.13 & 39.68 & 1 & 40.96 & 0.99 & 27.10 & 0.89 & 24.90 & 0.63 \\
\hline 4 & 40.05 & 1.02 & 39.15 & 1.11 & 59.52 & 1 & 61.44 & 1.00 & 40.65 & 0.89 & 37.35 & 0.61 \\
\hline 5 & 53.40 & 1.03 & 52.20 & 1.12 & 79.36 & 1 & 81.92 & 0.99 & 54.20 & 0.89 & 49.80 & 0.60 \\
\hline $\mathrm{RSD}(\%)$ & & 3.06 & & 1.31 & & & & 1.30 & & 2.42 & & 2.14 \\
\hline Average & & 1.00 & & 1.13 & & & & 1.00 & & 0.90 & & 0.61 \\
\hline
\end{tabular}

while the others had significant difference. The linearity data showed that the interception of impurities 2 and 4 were not less than 1 . This indicated that we couldn't neglect the influence of interception. The second one was only by one single concentration solution. But Table 4 clearly showed that the value of F1 increased with the increase of concentration, the change of F5 was exact contrary. F2 was more stable when the concentration was above $10 \mu \mathrm{g} / \mathrm{mL}$. The reason may relate with the extremely low absorption value of saponins. When the concentration of analytes was lower than $10 \mu \mathrm{g} / \mathrm{mL}$, the RSD would increase. Thus, we suggest that the correction factor should be applied in a relative stable range of concentrations, if the concentration of impurity 2 was too low in the bulk sample, the external standard method would be desirable.

\section{Ruggedness and robustness}

In the quality control of this natural product, the value of correction factor may change greatly if it was performed in different laboratories. Therefore, the stability of correction factor was investigated by ruggedness and robustness tests. In ruggedness test, five columns (three different Agilent TC-18 columns, Thermo ODS Hypersil and Diamonsil C18) were investigated. All these columns meet with the requirements of system suitability

Table 5 Results of ruggedness and robustness test

\begin{tabular}{llllll}
\hline & Imp-1 & Imp-2 & Imp-3 & Imp-4 & Imp-5 \\
\hline Diamonsil & 0.98 & 1.02 & 0.92 & 0.80 & 0.58 \\
Thermo & 1.08 & 1.12 & 1 & 0.9 & 0.77 \\
$\mathbf{2 0 6} \mathbf{~ n m}$ & 1.03 & 1.11 & 0.99 & 0.89 & 0.66 \\
$\mathbf{2 0 0} \mathbf{~ m m}$ & 1.02 & 1.07 & 0.99 & 0.89 & 0.72 \\
$\mathbf{3 5 ^ { \circ } \mathbf { C }}$ & 1.04 & 1.1 & 0.99 & 0.89 & 0.68 \\
$\mathbf{2 5} \mathbf{C}$ & 1.02 & 1.16 & 0.99 & 0.89 & 0.71 \\
$\mathbf{1 . 2} \mathbf{~ m l} / \mathbf{m i n}$ & 0.99 & 1.10 & 0.98 & 0.88 & 0.68 \\
$\mathbf{0 . 8} \mathbf{~ m l / m i n}$ & 1.03 & 1.16 & 0.99 & 0.90 & 0.67 \\
TC-C18 (1) & 0.99 & 1.12 & 1.00 & 0.90 & 0.63 \\
$\mathbf{T C - C 1 8 ~ ( 2 ) ~}$ & 1.02 & 1.12 & 0.99 & 0.90 & 0.62 \\
TC-C18 (3) & 1.00 & 1.13 & 1.00 & 0.90 & 0.61 \\
\hline
\end{tabular}

parameters which was shown in Table 3. In robustness tests, three related factors were studied, the temperature of the column $\left(25^{\circ} \mathrm{C}-35^{\circ} \mathrm{C}\right)$, the flow rate of the mobile phase $\left(0.8-1.2 \mathrm{~mL} \cdot \mathrm{min}^{-1}\right)$ and the UV detection wavelength $(200 \mathrm{~nm}-206 \mathrm{~nm})$. The results were shown in Table 5. On all Agilent TC-C18 columns, the mean correction factor of five impurities was 1.00, 1.12, 1.00, 0.90 and 0.62 , respectively. The result revealed that all the correction factors were stable on different Agilent TC-C18 columns. Besides, the value of F3 and F4 were stable when it was performed in different brands of chromatographic columns and modified experimental parameters, the reason may be the structure of Imp - 3 and Imp - 4 was very close to AR. Factors regarding the flow rate and column temperature have some influence on the Imp-2. When the flow rate was decreased from 1.2 to 0.8 , the correction factor of Imp-2 was increased from 1.10 to 1.16. Regarding column temperature, when the temperature was increased from $25^{\circ} \mathrm{C}$ to $35^{\circ} \mathrm{C}$, the correction factor of Imp-2 was decreased from 1.16 to 1.1. The possible reason may relate with the integration parameters, the peak area changed at the same integration parameters when there were small changes in experimental parameters.

\section{Analysis of three batches of clematichinenoside AR bulk samples}

The validated method was applied to quantitate impurities in three batches of AR bulk samples by using two methods: external standard method as method I, and single reference standard by five correction factors were used to determine the impurities of the same samples as method II. The sample solutions were prepared at $1 \mathrm{mg} / \mathrm{mL}$. The contents of impurities relative to AR were summarized in Table 6. The RSDs of sum contents of the five compound obtained by the two methods of each sample were less than $2 \%$. And the RSDs of Imp-1, Imp- 4, Imp-5 were more than 5\%. The reason might relate with the low concentration of impurities in samples. However, the difference can be neglected for quality control because the RSDs of sum contents meet the requirements. Therefore, single reference standard by correction factors could be used in the quality control of 
Table 6 The contents of clematichinenoside AR and its related impurities in three batches of bulk samples

\begin{tabular}{|c|c|c|c|c|c|c|c|c|c|}
\hline & \multicolumn{3}{|l|}{ Bulk-1 } & \multicolumn{3}{|l|}{ Bulk-2 } & \multicolumn{3}{|l|}{ Bulk-3 } \\
\hline & methodl & methodII & RSD & methodl & methodII & RSD & methodl & methodII & RSD \\
\hline Imp-1(\%) & 0.65 & 0.67 & 2.14 & 1.11 & 0.99 & 8.08 & - & - & - \\
\hline Imp-2(\%) & - & - & - & - & - & - & 2.47 & 2.36 & 3.22 \\
\hline clematichinenoside AR(\%) & 93.01 & - & - & 92.22 & - & - & 95.03 & - & - \\
\hline Imp-3(\%) & 4.38 & 4.57 & 3.00 & 4.17 & 4.34 & 2.83 & 2.08 & 2.12 & 1.35 \\
\hline Imp-4(\%) & 1.24 & 1.35 & 6.01 & 1.22 & 1.39 & 9.21 & - & - & - \\
\hline Imp-5(\%) & 1.02 & 0.89 & 9.63 & 1.03 & 0.94 & 6.46 & - & - & - \\
\hline Sum(\%) & 7.28 & 7.48 & 1.92 & 7.53 & 7.66 & 1.21 & 4.55 & 4.48 & 1.10 \\
\hline
\end{tabular}

related impurities. According to the regulation of State Food and Drug Administration, the content of AR should not be less than $90.0 \%$. So, all the samples comply with the limit.

\section{Conclusions}

The quality of bulk drug not only depends on the adopted procedure, but also on the side-reaction products, unreacted raw materials and intermediates, because they may render unwanted toxicological effects. Hence, thorough examination of related impurities plays a very important role in controlling the quality of bulk drug in the final product $[14,19]$. The impurities were isolated from AR bulk samples by column chromatography and semi-preparative HPLC methods. Five related impurities were characterized, including a new compound confirmed as $3-\mathrm{O}-\beta-[(\mathrm{O}-\alpha-\mathrm{L}-\mathrm{rhamnopyranosyl-}(1 \rightarrow 6)-\mathrm{O}-$ $\beta$-D-glucopyranosyl- $(1 \rightarrow 4)-\mathrm{O}-\beta$-D -glucopyranosyl- $(1 \rightarrow 4)$ O- $\beta$-D-ribopyra-nosyl- $(1 \rightarrow 3)-\mathrm{O}-\alpha-\mathrm{L}-$ rhamnopyranosyl$(1 \rightarrow 2)$ - $\alpha$-L-arabinopyranosyl)oxy] hederagenin $28-\mathrm{O}-\alpha-\mathrm{L}-$ rhamnopyranosyl-( $1 \rightarrow 4)-\mathrm{O}-\beta$-D-glucopyanosyl $-(1 \rightarrow 6)$ $\beta$-D- glucopyanosyl ester. Imp-1,3,4,5 were four known triterpenoid saponins isolated from Rhizoma Clematidis (Wei-Ling-Xian), Imp-2 (clematomandshurica saponin F) was a new triterpenoid saponins firstly found in AR bulk samples, it might be derived from the crude drug. In this paper, a simple gradient RP-HPLC method was developed and validated for the simultaneous determination of $\mathrm{AR}$ and its related impurities in bulk samples. By using experimental designs, optimum separation was successfully achieved and five impurities of AR could be separated in 20 minutes with satisfactory resolution, linearity, and sensitivity. Furthermore, two methods including external standard method and single reference standard with correction factor were applied to determine impurities in three batches of AR bulk samples. In order to ensure the accuracy of the determination, speed up the analysis, and reduce the cost of experimental procedure, the correction factor was better for the determination of related substances in a relative stable range of concentrations, which could be very helpful for the quality control of AR bulk sample and the safe clinical use in the future.

\section{Abbreviations \\ HPLC: High-performance liquid chromatography; ICH: International Conference on Harmonization; UV: Ultraviolet; LOD: Limit of detection; LOQ: Limit of quantification; SD: Standard deviation; RSD: Relative standard deviation; ELSD: Evaporative Light Scattering Detector; RP-HPLC: Reverse phase high-performance liquid chromatography; LC-MS/MS: Liquid chromatography-tandem mass spectrometry; TOF-MS: Time-of-flight mass spectrometry nuclear magnetic resonance; HMBC: Heteronuclear Multiple Bond Correlation; HR-ESI MS: High resolution electrospray ionization mass spectrometry; Rs: Resolution; As: Tailing factor; a: Selectivity; k': Capacity factor; NTP: Number of theoretical plates; AR: Clematichinenoside AR.}

\section{Competing interests}

The authors declare that they have no competing interests.

\section{Authors' contributions}

LFL planed and supervised the whole work, YZ carried out the experiments and analyzed the data statistically, YG, JS, XLZ and LY participated in writing the manuscript. All authors read and approved the final manuscript.

\section{Acknowledgements}

Financial support from the "The Creation for Significant New Drugs" a major scientific and technological initiative for "Eleventh Five-Year Plan" (No. 2009ZX09103-371), National Natural Science Foundation of China (Project No. 30772770) and Qing Lan Project (2010) are gratefully acknowledged.

Received: 24 September 2012 Accepted: 27 November 2012 Published: 8 December 2012

\section{References}

1. The State Pharmacopoeia Commission of The People's Republic of China: Pharmacopoeia of the People's Republic of China. China Medical Science 2010, Volume 1:234.

2. Shi $S$, Jiang D, Zhao $M$, et al: Preparative isolation and purification of triterpene saponins from Clematis mandshurica by high-speed countercurrent chromatography coupled with evaporative light scattering detection. J Chromatogr B 2007, 852:679-683.

3. Mimaki Y, Yokosuka A, Hamanaka M, et al: Triterpene saponins from the roots of Clematis chinensis. J Nat Prod 2004, 67:1511-1516.

4. Shi S, Jiang D, Dong C, et al: Triterpene saponins from Clematis mandshurica. J Nat Prod 2006, 69:1591-1595.

5. Liu L, Ma X, Wang YX, et al: Triterpenoid saponins from the roots of Clematis chinensis Osbeck. J Asian Nat Prod Res 2009, 11:389-396.

6. Wu W, Xu X, Dai $Y$, et al: Therapeutic effect of the saponin fraction from clematis chinensis osbeck roots on osteoarthritis induced by monosodium iodoacetate through protecting articular cartilage. Phytother Res 2010, 24:538-546.

7. Fu Q, Zan K, Zhao M, et al: Triterpene saponins from clematis chinensis and their potential anti-inflammatory activity. J Nat Prod 2010, 73:1234-1239. 
8. Peng C, Perera PK, Li Y, et al: Anti-inflammatory effects of Clematis chinensis Osbeck extract(AR-6) may be associated with NF-kB, TNF-a, and COX-2 in collagen-induced arthritis in rat. Rheumatol Int 2012, 32:3119-3125.

9. Lu X, Fang W, Li Y, et al: Analgesic and anti-inflammatory effect of clematichinenoside. Chinese Journal of New Drugs 2009, 18:1535-1539.

10. Sun $S, L i$ Y, Fang W, et al: Effect and mechanism of AR-6 in experimental rheumatoid arthritis. Clin Exp Med 2010, 10:113-121.

11. Dong C, Liu L, Li M, et al: The preparation and preliminary pharmacodynamics of clematichinenoside microemulsion. Chinese Traditional Patent Medicine 2011, 33(7):1159-1163.

12. $M a X, X i e ~ L, ~ L i u ~ L$, et al: Simultaneous quantification of seven main triterpenoid saponins in Radix et Rhizoma Clematidis by LC-ELSD. Chromatographia 2009, 69:437-443.

13. Wang D, Li F, Li P, et al: Validated LC-MS/MS assay for the quantitative determination of clematichinenoside $A R$ in rat plasma and its application to a pharmacokinetic study. Biomed Chromatogr 2012, 26(10):1282-1285.

14. ICH: Q3A Impurities in New Drug Substances. In Proceedings of the international conference on harmonization. 2006:1-22.

15. Kizu H, Shimana H, Tommimori T: Studies on the constituents constituents of Clematis Species IV. The Constituents of Clematis stans Sieb. Et Zucc. Chem Pharm Bull 1995, 43(12):2187-2194.

16. Shao $B$, Qin $G, X u R$, et al: Triterpenoid saponins fromClematis chinensis. Phytochemistry 1995, 38(6):1473-1479.

17. ICH: Q1A Stability Testing of New Drug Substances and Products. In Proceedings of the international conference on harmonization. 2003:1-15.

18. Hou J, Wua W, Da J, et al: Ruggedness and robustness of conversion factors in method of simultaneous determination of multi-components with single reference standard. J Chromatogr A 2011, 1218:5618-5627.

19. ICH: Q3B Impurities in New Drug Substances. In Proceedings of the international conference on harmonization. 2006:1-12.

doi:10.1186/1752-153X-6-150

Cite this article as: Zhou et al: Development and validation of a

chromatographic method for determining Clematichinenoside AR and related impurities. Chemistry Central Journal 2012 6:150.

\section{Publish with ChemistryCentral and every scientist can read your work free of charge \\ "Open access provides opportunities to our colleagues in other parts of the globe, by allowing anyone to view the content free of charge." \\ W. Jeffery Hurst, The Hershey Company. \\ - available free of charge to the entire scientific community \\ - peer reviewed and published immediately upon acceptance \\ - cited in PubMed and archived on PubMed Central \\ - yours - you keep the copyright \\ Submit your manuscript here: \\ http://www.chemistrycentral.com/manuscript/<smiles>c1ccccc1</smiles> \\ Chemistry Central}

